Ingeniería Investigación y Tecnología, ISSN 2594-0732,

IX. 2. 149-159, 2008 (artículo arbitrado)

DOI: http://dx.doi.org/10.22201/fi.25940732e.2008.09n2.012

\title{
DETECCIÓN del dAÑO geNERAdO EN MATERIALES COMPUESTOS dE MATRIZ POLIMÉRICA EMPIEANdO LA TÉCNICA dE EMISIÓN ACÚSTICA
}

\author{
DAMAGE dETECTION ON POLYMERIC MATRIX COMPOSITE MATERIALS \\ DY USING ACOUSTIC EMISSION TECHNIQUE
}

\author{
J. Cauich-Cupul, J.A. Rodríguez-Laviada y C.R. Ríos-Soberanis \\ Centro de Investigación Científica de Yucatán, \\ Unidad de Materiales Mérida, Yucatán, México \\ E-mail: rolando@cicy.mx
}

(Recibido: agosto de 2006; aceptado: octubre de 2007)

\begin{abstract}
Resumen
Con el objeto de predecir el comportamiento mecánico de un material compuesto durante su vida de senvicio es importante monitorear el inicio y desarrollo de las fallas y los efectos producidos por los distintos medios de degradación, además la aparición de grietas está relacionada con la integridad estructural del componente y su vida durante la fatiga. Ésta, entre otras razones, ha motivado el empleo de técnicas no destructivas, tales como la emisión acústica (EA) para el análisis intrínseco de las propiedades mecánicas de un material. El método EA, ha demostrado excelentes resultados en la detección e identificación de sitios de iniciación, propagación y desarrollo de fallas y mecanismos de fractura de materiales compuestos manufacturados con matriz polimérica, asimismo, la posibilidad de relacionar los modos de fractura con la geometría del refuerzo. El objetivo de este trabajo es remarcar la importancia de la emisión acústica como herramienta para detectar los parámetros mecánicos al caracterizar los materiales en respuesta a los esfuerzos externos y en procesos de degradación. Los resultados obtenidos en diversos estudios son presentados para apoyar la investigación en la detección del daño en los composites, de esta manera, involucrar totalmente los beneficios de la emisión acústica en la caracterización de materiales compuestos.
\end{abstract}

Descriptores: Materiales compuestos, mecanismos de fractura, emisión acústica.

\begin{abstract}
In order to predict the mechanical behaviour of a composite during its service life, it is important to study the initiation and development of cracks and its effects induced by degradation. The onset of damage is related to the structural integrity of the component and its fatigue life. For this, among other reasons, non-destructive techniques have been widely used nowadays in composite materials characterization such as acoustic emission (AE). This method has demonstrated excellent results on detecting and identifying initiations sites, cracking propagation and fracture mechanisms of polymer matrix composite materials. At the same time, mechanical behaviour has been related intimately to the reinforcement architecture. The goal of this paper is to remark the importance of acoustic emission technique as a unique tool for characterising mechanical parameters in response to external stresses and degradation processes. Some results obtained from different analysis are discussed to support the significance of using $A E$, technique that will be increased continuously in the composite
\end{abstract}


DOI: http://dx.doi.org/10.22201/fi.25940732e.2008.09n2.012

Detección del daño generado en materiales compuestos de matriz polımérica ...

materials field due to its several alternatives for understanding the mechanical behaviour, therefore the objective of this manuscript is to involve the benefits and advantages of $A E$ in the materials characterization.

Keywords: Composite materials, mechanisms of fracture, acoustic emission.

\section{Introducción}

La técnica de emisión acústica (EA) está basada en la generación de ondas acústicas (esfuerzo elástico o de presión) por el proceso de la rápida propagación de una microfalla u otro tipo de fuentes de energía mecánica. Sensores piezoeléctricos altamente sensibles detectan la señal emitida desde el punto inicial por movimiento superficial dinámico y la convierten en una señal eléctrica. La frecuencia general utilizada es entre $50 \mathrm{kHz}-1$ $\mathrm{MHz}$. frecuencias mas bajas están relacionadas con ruido externo. La técnica de emisión acústica es una medida del tiempo real y de evaluación en línea. Básicamente proporciona información tal como: "cuándo" (tiempo, parámetro de carga externa), "cuánto" (proporción), "intensidad” (amplitud, energía) y "dónde" (localización de las fuentes de $E A)$, ocurren las ondas de energía mecánica que producen los procesos de daño en diversos tipos de materiales estructurales. Si existe un desarrollo del defecto en el cuerpo, la emisión acústica revelará su existencia durante su crecimiento. Esta es una de las principales ventajas de la emisión acústica "Monitoreo en Tiempo Real". Si alguna falla sucede o se propaga, la tecnología de EA evalúa la posibilidad del desarrollo de grietas al detectar las ondas elásticas que son generadas en el material, daños que no son audibles ni visibles al ser humano. En los últimos años la demanda del empleo de la tecnología EA ha aumentado para su aplicación en materiales compuestos estructurales fibroreforzados, debido a la gran ventaja que implica el estudio directo en línea de un material sometido a diversos esfuerzos externos. Sin embargo, debido a la heterogeneidad de los materiales compuestos, los procesos de fractura son extremadamente complejos. La emisión acústica ha probado ser un excelente método de caracterización no destructiva (pasiva) para el control del daño y la inspección periódica en senvicio de materiales compuestos fibroreforzados basados en matrices plásticas. Sus ventajas lo han llevado al establecimiento de diversos procedimientos estándares para evaluar la importancia de los defectos identificando los sitios de inicio, la propagación y el desarrollo de las fallas hasta la fractura. La figura 1 presenta un típico equipo de emisión acústica en el cual la señal es captada y registrada en el ordenador donde es posible realizar las combinaciones pertinentes de la información obtenida. Parte importante de la función del equipo EA son los sensores, de los cuales, el elemento activo de la mayoría de éstos es un cerámico piezoeléctrico que convierte la energía eléctrica a acústica y viceversa. Muchos factores incluyendo el material, su diseño mecánico y eléctrico, así como las condiciones de carga externa mecánica y eléctrica, influyen en su resultado. Durante la adhesión del sensor en la superficie del material a ensayar y con el objeto de evitar pérdidas de las señales elásticas durante la transmisión de un medio a otro, un agente de acoplamiento debe depositarse como interfase entre la superficie del sensor y la muestra. Éste puede ser desde agua hasta algún tipo de grasa (i.e. vaselina); sin embargo, se recomienda un medio denso y viscoso.

La respuesta de la señal acústica es sin duda de las más versátiles debido a la amplia variedad de parámetros que puede registrar. La figura 2 presenta una gráfica señalando los parámetros obtenidos a través de la técnica de emisión acústica, donde es posible relacionar las señales detectadas con los mecanismos de fractura de un material identificando la generación de fallas tales como microgrietas, delaminación de la interfase fibra/resina, la fractura de la matriz y finalmente de las fibras. 
J. Cauich-Cupul, J.A. Rodríguez-Laviada y C.R. Ríos-Soberanis

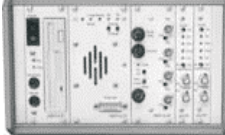

Figura 1. Instrumentación para la aplicación de la técnica de la emisión acústica

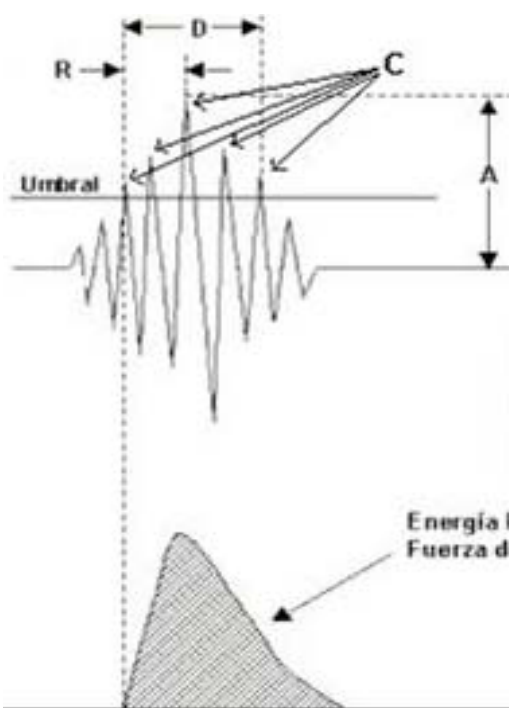

Figura 2. Señal y parámetros acústicos

Varios investigadores como Ativitavas et al. (2006), Sodomía (2004) y Valea et al. (2002), han empleado la técnica de la emisión acústica como método alternativo para la caracterización de materiales, demostrando su capacidad como herramienta básica en el estudio del comportamiento mecánico de materiales compuestos; sin embargo, su empleo aún es limitado debido al desconocimiento de las bondades de esta técnica tan valiosa. Gao et al. (1999) determinaron la relación existente entre la generación de grietas y la arquitectura de un refuerzo textil tipo petatillo (woven) 
en matriz epóxica empleando la emisión acústica como medio para relacionar los eventos (señales que exceden el valor mínimo del umbral) con la curva de esfuerzo-deformación. Sin lugar a dudas, esta técnica ha permitido relacionar fuentes de iniciación de fallas para ser relacionados con el comportamiento observado en las curvas de esfuerzo-deformación con el objeto de atribuir las diferentes etapas del proceso del daño producido al efecto del arreglo de las fibras en el material de refuerzo. Tal como se ha mencionado anteriormente, el objetivo de este artículo es remarcar los grandes beneficios que se obtienen con la caracterización mecánica de materiales compuestos a través de la técnica de la emisión acústica, por lo tanto, a continuación se discutirán brevemente algunos de los avances obtenidos en investigaciones recientes.

\section{Identificación de mecanismos de fractura en compositos reforzados con textiles}

La relación entre la arquitectura del textil y la acumulación del daño bajo carga en tensión ha sido investigado par varios autores (Gao et al., 1999), (Ramakrishna et al., 1994) y (Bohse, 2004). Por otro lado, en este trabajo de investigación la téc- nica de la emisión acústica ha sido empleada para apoyar el análisis del comportamiento mecánico por efecto de la geometría del tejido. En este caso, la relación fue investigada para un material compuesto reforzado con tejido tipo "knitted" Milano 2x68 Tex de fibra de vidrio "E" (Figura 3) orientado a diferentes ángulos con respecto a la dirección de la carga. Con el objeto de observar el daño progresivo, el textil de refuerzo fue embebido en resina epóxica (matriz polimérica), de esta manera, los laminados transparentes finales permitieron el monitoreo del desarrollo de las fallas en el material compuesto en función del incremento de la deformación, permitiendo una observación directa de la secuencia del daño. El textil tipo "knitted" está constituido de interconexiones de secciones circulares de aglomeraciones de fibras, que conforman sus dos direcciones principales: a lo largo del textil, refiriéndose a la orientación a $0^{\circ}$ (entramado en la dirección longitudinal), y a lo ancho, identificándose la orientación a 90 (entramado en la dirección transversal).

Para la manufactura de estos laminados se empleó un sistema de resina epóxica como matriz por el proceso de apilado en húmedo (wet lay up). Las probetas para ensayos mecánicos fueron

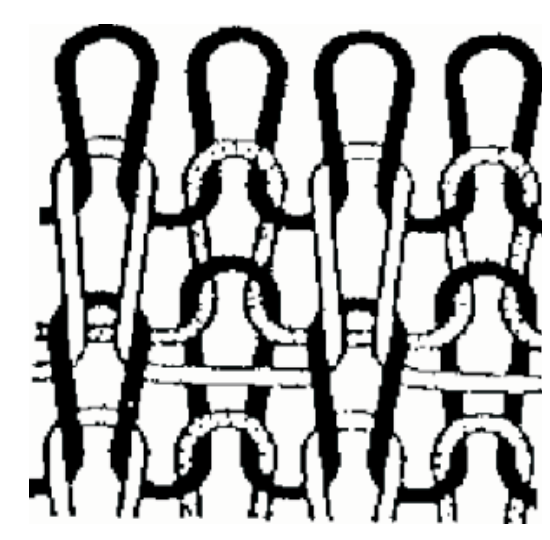

Figura 3. Estructura milano: dos secciones simples de fibras tejidos en dos juegos separados de agujas (trama 2 y 3), los cuales están sujetos por una hilera 1x1 (trama 1)

152 Ingeniería Investigación y Tecnología, ISSN 2594-0732 
J. Cauich-Cupul, J.A. Rodríguez-Laviada y C.R. Ríos-Soberanis

obtenidas de los laminados con las dimensiones presentadas en la figura 4. Dos galgas extensométricas, una longitudinal y otra transversal fueron adheridas al centro de las probetas para obtener los parámetros mecánicos en ambas direcciones. Así mismo, dos sensores de emisión acústica fueron colocados con el objeto de captar la señal emitida durante el proceso de fractura.

Un sistema de emisión acústica (AECL 2100.M) fue utilizado para monitorear las ondas mecánicas provocadas como resultado de la generación y crecimiento de defectos en el material. Los sensores se adhirieron a la superficie de la muestra con cinta adhesiva empleando vaselina como agente acoplante. La mayoría del ruido externo tiene picos de amplitud menores a $40 \mathrm{db}$ (Ramakrishna et al., 1994), dato a tomar en cuenta para establecer el punto del umbral de la señal acústica. Análisis preliminares fueron realizados para establecer el valor apropiado del umbral, el cual se consideró $0.6 \mathrm{~V}$. permitiendo al sensor colectar sólo aquellos eventos cuya señal excediera este valor, considerando como ruido externo el resto. Resultados arrojaron información acerca del comportamiento y de la respuesta mecánica sobre la geometría del textil de acuerdo a la dirección en que la carga era sometida, los cuales están íntimamente relacionados. El desarrollo de las fallas dependiendo de la orientación del tejido con la dirección del esfuerzo aplicado es presentado en la figura 5 , donde es posible observar la diferencia en el progreso de las fisuras. A $0^{\circ}$ existe un patrón establecido por el tejido de tal manera que las grietas se propagan a espacios específicos relacionados con el entramado. Cuando el tejido es orientado a $45^{\circ}$ las grietas intentan continuar un patrón diagonal cada $4 \mathrm{~mm}$ también dictado por la arquitectura del textil y, finalmente a un ángulo de $90^{\circ}$ las fallas se desarrollan y propagan completamente al azar sin establecer un patrón constante. Es importante establecer que debido a la mayor concentración de fibras orientadas a $0^{\circ}$, esta dirección presenta mejores propiedades mecánicas que su contraparte transversal a $90^{\circ}$.

Típicas curvas de esfuerzo-deformación-emisión acústica de los materiales en las tres respectivas orientaciones son presentadas en la figura 6. En ellas, es posible observar la correlación existente entre la curva y la señal acústica, la cual detecta microdaño en el material previo a la aparición de fallas visibles o audibles al ser humano. De la misma forma, la intensidad de la señal esta íntimamente relacionada con la discontinuidad (hombros) de la curva de esfuerzo-deformación. Además, la emisión acústica exhibe información importante relacionada con la resistencia del material. En la figura 6 es posible comprobar que el material orientado a $0^{\circ}$ es mas resistente, debido a que las señales acústicas originadas por la aparición de las grietas son detectadas aproximadamente a $0.87 \%$ de deformación, para el material orientado a $45^{\circ}$ las microfallas inician a $0.68 \%$ indicando una resistencia menor, y finalmente a $90^{\circ}$ el daño inicia ligeramente arriba del anterior, aproximadamente a $0.72 \%$. Este comportamiento es a causa de que las direcciones a $0^{\circ}$ y $90^{\circ}$ se encuentran una mayor cantidad de fibras orientadas paralelamente a la carga aplicada. La primera

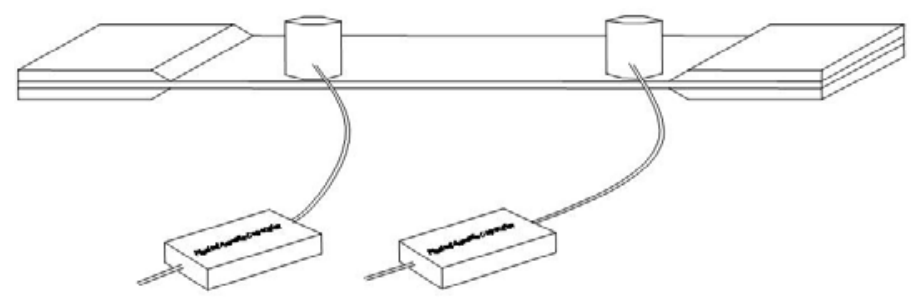

Figura 4. Dimensiones de las probetas 
comparación que puede obtenerse sobre la iniciación y propagación del daño para varios ángulos es presentada en la tabla 1, la cual exhibe la iniciación del predaño registrado por la señal acústica inaudible e invisible a simple observación y el primer agrietamiento de la matriz el cual se aprecia visualmente como daño significativo y apoya las observaciones anteriores de resistencia del material a los esfuerzos externos, obviamente por efecto de la geometría del textil.

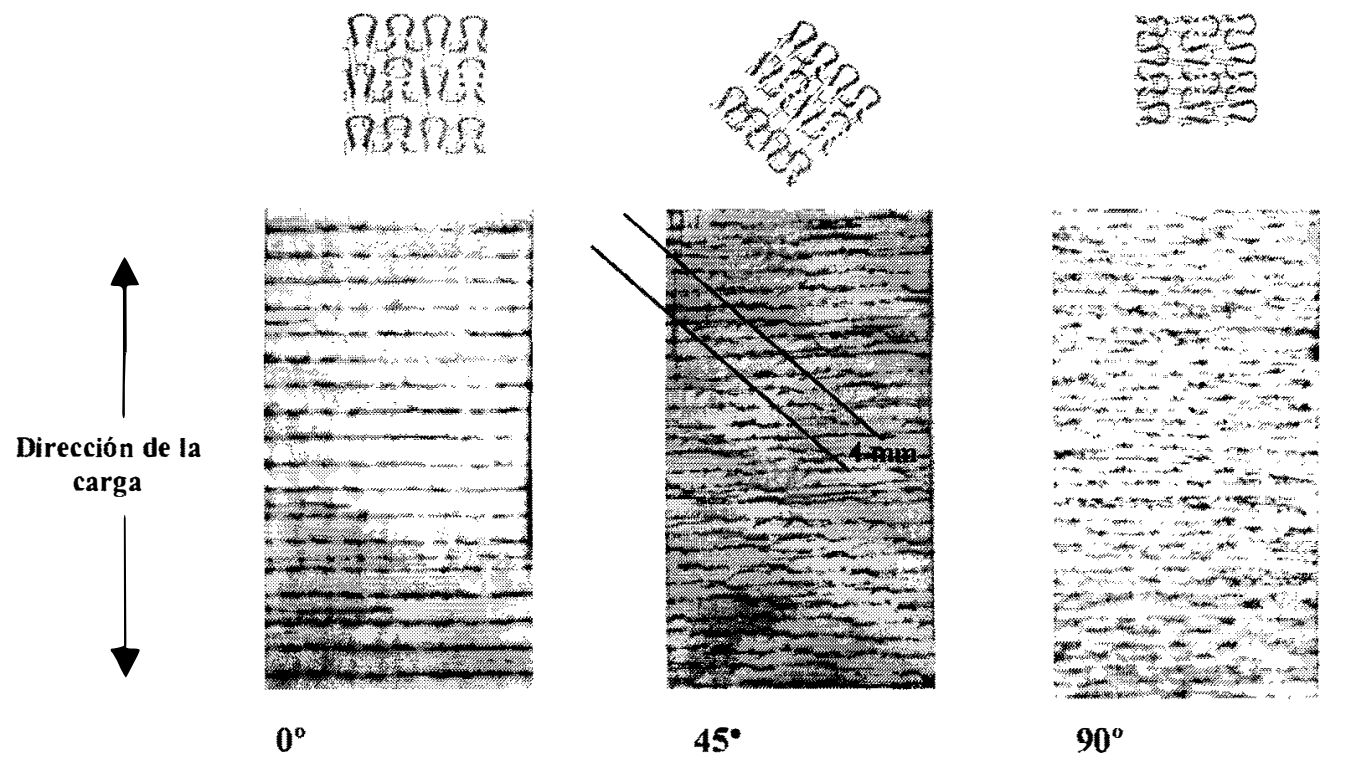

Figura 5. Respuesta mecánica del material compuesto dependiendo de la orientación del textil

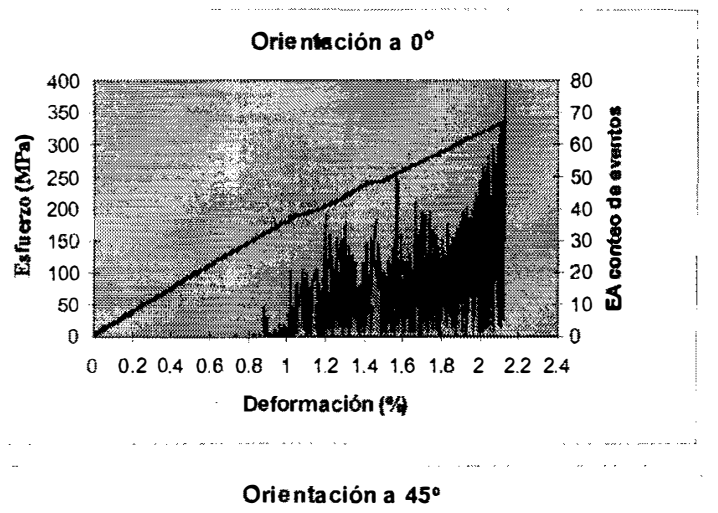

(a)

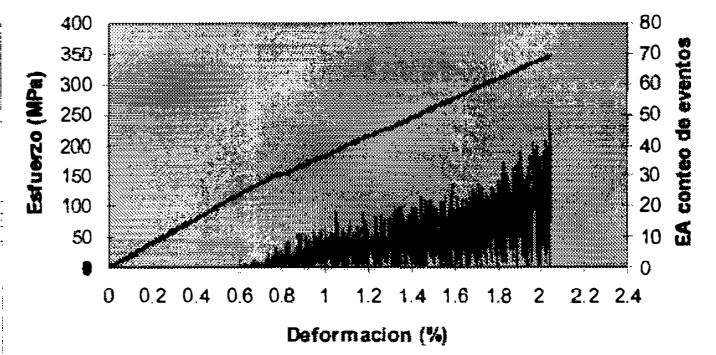

(b)

Figura 6. Curvas de esfuerzo-deformación-EA de probetas a: a) $0^{\circ}$, b) $45^{\circ}$ y c) $90^{\circ}$ (continúa ...) 


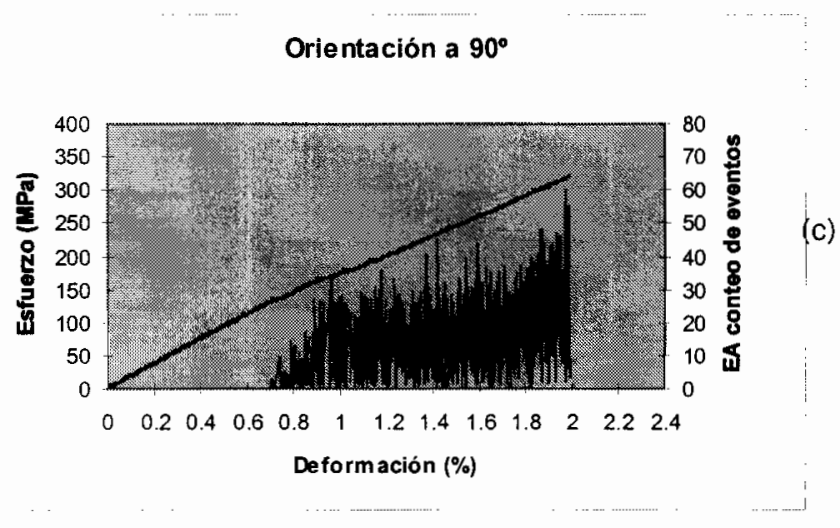

Figura 6. Curvas de esfuerzo-deformación-EA de probetas a: a) $0^{\circ}$, b) $45^{\circ}$ y c) $90^{\circ}$ (... continuación)

Tabla 1. Variación de datos tomados de la señal de emisión acústica

\begin{tabular}{ccc}
\hline Orientación & $\begin{array}{c}\text { Predaño } \\
\text { (\% elongación) }\end{array}$ & $\begin{array}{c}\text { Inicio de las grietas } \\
\text { (\% elongación) }\end{array}$ \\
\hline $0^{\circ}$ & $0.80-0.95$ & $0.95-1.05$ \\
$45^{\circ}$ & $0.65-0.70$ & $0.75-0.85$ \\
$90^{\circ}$ & $0.65-0.85$ & $0.80-0.90$ \\
\hline
\end{tabular}

Probetas seleccionadas fueron ensayadas a tensión hasta el esfuerzo en el cual se registraban las primeras señales de emisión acústica (antes de la aparición de las grietas visibles denominado predaño). Se tomaron diversas piezas de estas muestras y se realizó un meticuloso proceso de pulido con la intención de observar la superficie al microscopio. En estas muestras se determinaron los sitios de iniciación de la falla. Estos sitios de mag- nificación de esfuerzos se encontraron en los puntos donde las mechas se cruzan (cross-over points), señalados en la figura 7. Así se estableció la existencia de delaminación en la interfase fibra/ matriz como causa inicial de la formación del daño en el material compuesto.

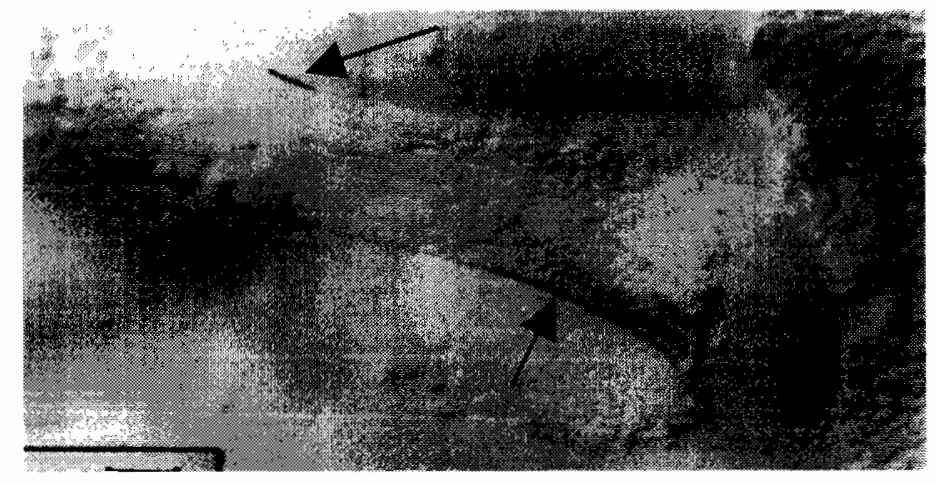

Figura 7. Sitios de iniciacoón de fallas detectados con la emisión acústica 
Detección del daño generado en materiales COMPUESTOS de matriz polıMÉrica ...

Las fracciones volumétricas fueron obtenidas para todos los laminados utilizando la técnica de la incineración de la matriz, la cual consiste en depositar una muestra de aproximadamente $20 \mathrm{~mm} x$ $20 \mathrm{~mm}$ previamente pesados, en crisoles de porcelana de peso conocido y llevados a incineración en una mufla a $600^{\circ} \mathrm{C}$ durante $3 \mathrm{hrs}$. Después de este tiempo, la resina ha sido eliminada por completo y el crisol es enfriado a temperatura ambiente. Una vez enfriado, el crisol es pesado nuevamente y obtenido el resultado por gravimetría. La densidad del vidrio $\left(\rho_{\mathrm{f}}\right)$ y la resina $\left(\rho_{\mathrm{m}}\right)$ es 2.56 $\mathrm{g} / \mathrm{cm}^{3}\left(2560 \mathrm{~kg} / \mathrm{m}^{3}\right)$ y $1.21 \mathrm{~g} / \mathrm{cm}^{3}\left(1210 \mathrm{~kg} / \mathrm{m}^{3}\right)$ respectivamente. La fracción volumétrica fue calculada usando la ecuación 1.

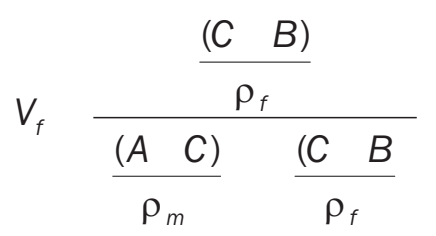

donde A es la masa del crisol y la muestra original, B es la masa del crisol, $C$ es la masa del crisol y el residuo de fibras, $\rho_{f}$ es la densidad de las fibras y $\rho_{\mathrm{m}}$ es la densidad de la matriz. Para todos los laminados la fracción volumétrica promedio fue obtenido a un valor de $29.0 \pm 0.2 \%$.

\section{Caracterización de la velocidad de propagación en resina por emisión acústica}

Un factor que puede influir disminuyendo la resistencia de la interfase fibra-matriz de un material compuesto de matriz polimérica es la presencia de humedad en los constituyentes. El problema de absorción de humedad en resinas epóxicas es de gran importancia, ya que cuando son expuestas a la humedad o a un medio ambiente húmedo, típicamente llegan a absorber de 1 a $7 \%$ de agua en peso. Esta humedad absorbida tiene un efecto deteriorante en las propiedades físicas de la resina epóxica. Se sabe que el factor más importante que propicia esta ganancia en peso de agua es la polaridad de los grupos funcionales de la resina epóxica. Por lo tanto, es importante el análisis de la respuesta de un sistema de resina ante la degradación de humedad.

Esta investigación se desarrolló empleando una resina epóxica basada en diglicidil éter de bisfenol A (DGEBA), con la cual se elaboraron probetas para realizar ensayos de tensión. Previamente estas probetas fueron introducidas en desecadores acondicionados a 25\%, 55\%, y $95 \%$ de humedad relativa con el objeto de medir inicialmente el porcentaje de humedad absorbido. En este caso, la técnica de emisión acústica fue utilizada para calcular la velocidad de propagación de la onda mecánica entre dos puntos de distancia conocida, teniendo como punto de referencia una muesca manualmente elaborada de $0.5 \mathrm{~mm}$ de profundidad con el objeto de funcionar como concentrador de esfuerzos para la generación y propagación de una fisura. La velocidad de propagación fue determinada con la diferencial de los tiempos de retraso $(\Delta t)$, que se registraron con las señales del sensor 1 y el sensor 2 al fracturarse la muestra por completo. La velocidad de propagación se calculó para cada porcentaje de humedad relativa. La figura 8 presenta las dimensiones y características de las probetas, incluyendo los sensores de emisión acústica.

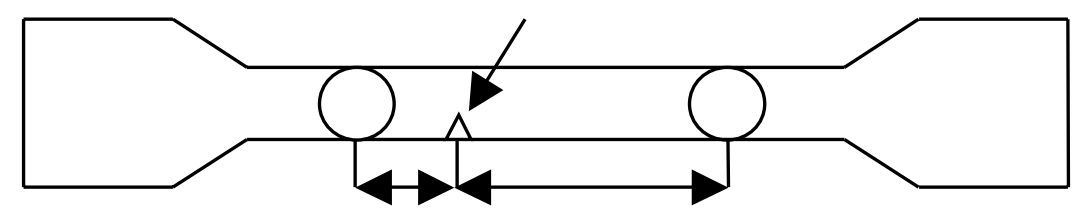

Figura 8. Esquema de las probetas para emisión acústica 
La velocidad de propagación de la señal se determinó por medio de la ecuación 2:

$$
\begin{aligned}
& \Delta d \quad V \Delta t \\
& V \quad \Delta d / \Delta t
\end{aligned}
$$

Donde:

V es la velocidad de onda de la señal,

$\Delta d$ es la separación de la muesca al sensor,

$\Delta t$ es la diferencial de tiempo entre los sensores.

La figura 9 muestra el comportamiento que sigue la velocidad de propagación de la señal acústica, cuando la resina epóxica ha sido expuesta a tiempos prolongados de humedad relativa del $95 \%$. Se observa que a una absorción del $2 \%$ de humedad, la velocidad de propagación de la señal acústica en el material, disminuye aproximadamente un $22.72 \%$, como resultado del efecto de la plastificación de la matriz. La velocidad de propagación de la señal acústica depende en gran parte del medio en la que se origine la señal, y de las características físico-químicas del material en la que se realice la prueba acústica. La velocidad de propagación de la señal acústica en un material rígido es rápida e intensa, en comparación con el material que presenta una disminución en sus propiedades de rigidez, en estos materiales la velocidad de propagación de la señal acústica es menor y menos intensa. En el caso de la resina epóxica, después de estar sometida a condiciones de humedad relativa controlada, cambian las propiedades del módulo elástico de un material rígido a un material menos rígido ocasionado por la plastificación; debido a este fenómeno, la velocidad de propagación acústica se reduce por la amortiguación molecular que se origina.

\section{Conclusiones y discusiones}

Tal y como se ha demostrado con los resultados anteriores, la tecnología de emisión acústica es un método muy poderoso de detección en línea y de análisis de procesos de fractura activos relacionados con la matriz, fibra e interfase de materiales compuestos. En el primer caso EA indica el valor de esfuerzo y deformación en el cual inicia el proceso de fallas, aún cuando éstas no son significativas, es decir, no son detectadas en la curva de

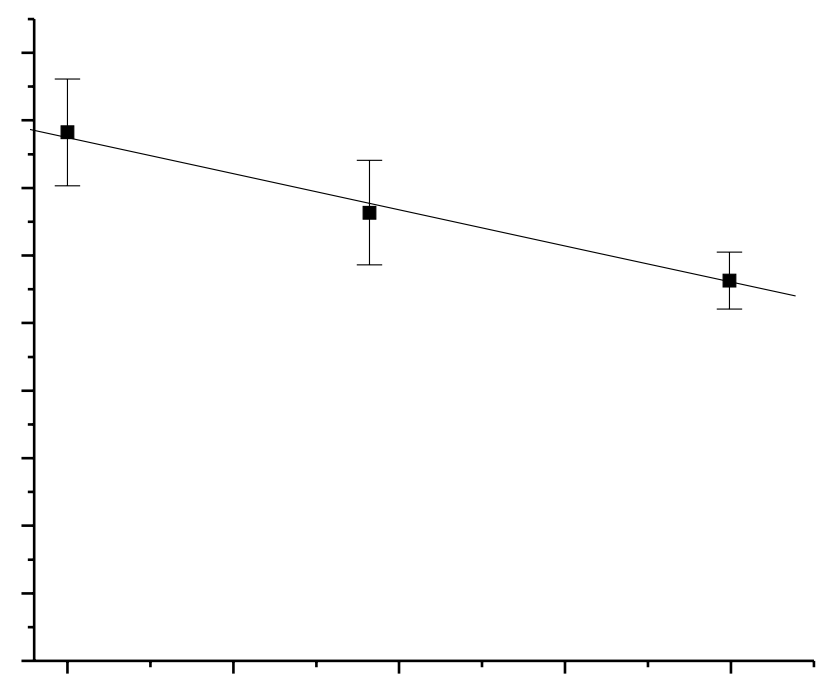

Figura 9. Gráfica de la determinación de la velocidad de propagación de la señal acústica 
Detección del daño generado en materiales COMPUESTOS de matriz polimérica ...

esfuerzo-deformación ni visiblemente (denominado predaño). Al obtener este valor y a través del proceso microscópico, es posible identificar la zona de magnificación de esfuerzos, y por ende, los sitios de iniciación. Además, los mecanismos de fractura del material compuesto son relacionados con la geometría del refuerzo. En el segundo caso se exhibe la degradación de la resina epóxica por efecto de la plastificación producida por la absorción del agua. La señal de EA detecta la atenuación de la velocidad del sonido por efecto de la degradación.

Varios proyectos (Prosser et al., 1995) y (Takuma, 2004) ahondan sus investigaciones tratando de descifrar el proceso y desarrollo de fisuras y fallas en composites, lo cual es un trabajo laborioso, debido a la diversidad de modos de fallas que presentan esta clase de materiales donde factores tales como la naturaleza de la matriz, el tipo y geometría del refuerzo, la interfase, los filamentos, las mechas, etc., afectan de manara distinta su comportamiento mecánico. Asimismo, tal como ya se ha presentado, a través de las ondas acústicas es posible determinar la velocidad y la atenuación producida por cambios físicos en diferentes materiales, con lo cual es posible analizar la degradación. De esta manera se demuestra la versatilidad de esta técnica.

\section{Referencias}

Ativitavas N., Pothisiri T. and Fowler T.J. Identification of fiber-reinforced plastic failure mechanisms from acoustic emission data using neural net- works. Journal of Composite Materials, 40:193- 226. 2006

Bohse J. Damage analysis of polymer matrix composites by acoustic emission testing. European Working Group Acoustic Emission (EWGAE 26 ${ }^{\text {th }}$ ). Berlin. Septiembre. 2004.

Gao F., Boniface L., Ogin S.L., Smith P.A. and Greaves R.P. Damage accumulation in wovenfabric CFRP laminates under tensile loading: Part 1. Observations of damage accumulation. Composite Science and Technology, 59:123136. 1999.

Prosser W.H., Jackson K.E., Kellas S., Smith B.T., McKeon J. and Friedman A. Advanced, waveform based acoustic emission detection of matrix cracking composites. Materials evaluation, 53: 1052-1058, 1995.

Ramakrishna S. y Hull D. Tensile Behaviour of Knitted Carbon-Fibre Fabric/Epoxy Laminates. Part I: Experimental. Journal of Composites Science and Technology, 50: 237-247, 1994.

Sodomka L. Application of acoustical emission as a new effective diagnostic tool in the textile fields. European Working Group Acoustic Emission, (EWGAE 26 ${ }^{\text {th }}$ ). Berlin. Septiembre. 2004.

Takuma M. and Shinke N. Evaluation of fatigue damage for FRM with $A E$ method. $17^{\text {th }}$ International Acoustic Emission Symposium (IAES-17). Tokio, Japón. Noviembre. 2004.

Valea-Pérez A., Martínez-Jequier J., MondragónEgaña I. y González-Arce M.L. Aplicación de la emisión acústica al estudio de la fractura de un material composite de matriz vinilester. Anales de Mecánica de la Fractura, 19:257-262, 2002. 
DOI: http://dx.doi.org/10.22201/fi.25940732e.2008.09n2.012

J. Cauich-Cupul, J.A. Rodríguez-Laviada y C.R. Ríos-Soberanis

\section{Semblanza de los autores}

Javier Cauich-Cupul. Obtuvo el título de ingeniero mecánico en el Instituto Tecnológico de Mérida en el año de 1994. Formó parte del Centro de Investigación Científica de Yucatán (CICY) en 1995. Cursó los estudios de maestría en materiales poliméricos en el CICY logrando el grado en 2004. Desde entonces, obtuvo la categoría de técnico titular A en la Unidad de Materiales en el área de mecánica. A lo largo de su carrera ha trabajado en colaboración con investigadores en diversos proyectos relacionados con materiales compuestos y ha realizado servicios en conjunto al sector privado. Su línea de investigación se enfoca al desarrollo de materiales compuestos fibroreforzados y su caracterización mecánica. Ha publicado con algunos investigadores en revistas arbitradas nacionales e internacionales del área científica con importancia en materiales compuestos.

José de los Ángeles Rodríguez-Laviada. Obtuvo el título como ingeniero químico por el Instituto Tecnológico de Mérida (ITM) en 1994. Asimismo, la maestría en ciencias en ingeniería química en 2006 por el Centro Universitario de Ciencias Exactas e Ingenierías (CUCEI, UdG). Forma parte de investigación en la Unidad de Materiales del Centro de Investigación Científica de Yucatán (CICY) desde el 2004. Sus campos de interés son el desarrollo de materiales compuestos reforzados con fibras sintéticas y naturales.

Carlos Rolando Ríos-Soberanis. Obtuvo su licenciatura en química industrial en el año de 1992 por la Facultad de Ingeniería Química Industrial de la Universidad Autónoma de Yucatán (UADY). Realizó satisfactoriamente sus estudios de posgrado en la Universidad de Surrey, Inglaterra, en donde recibió la maestría en tecnología de materiales avanzados en 1998, así como el grado de doctor (PhD) en 2002. Ingresó al Centro de Investigación Científica de Yucatán en enero de 2002 como investigador en la Unidad de Materiales donde su principal línea de investigación es la manufactura y caracterización física, química y mecánica de materiales compuestos reforzados con fibras sintéticas y naturales en forma de textiles con arquitectura definida. Ha publicado en las principales revistas del área de composites y ha presentado conferencias en diversos países como Francia, España, Inglaterra, Japón, USA y México entre otros. Pertenece al sistema Nacional de Investigadores desde 2003. Es miembro de la Society of Polymers Engineering (SPE) desde 2004 y de la Acoustic Emission Working Group (AEWG) desde 2005. 